\title{
Indeterminacy in the Small Open Economy Ramsey Growth Model
}

\author{
Mark Weder* \\ Department of Economics \\ Humboldt University Berlin \\ Spandauer Str.1 \\ 10178 Berlin \\ Germany
}

March 16, 1999

\begin{abstract}
This paper constructs a small open economy version of the two sector Benhabib-Farmer (1996) indeterminacy model. It can be shown that sunspot equilibria arise at significantly lower magnitudes of increasing returns to scale than in the original closed economy model. Furthermore, if a mix of externalities across sectors is admitted, indeterminacy can be obtained even with decreasing aggregate returns to scale.
\end{abstract}

${ }^{*}$ I have benefitted from discussions with Jess Benhabib, Michael Burda, Amartya Lahiri and Andreas Ruppin. All remaining errors are my own. A research grant from the Deutsche Forschungsgemeinschaft is gratefully acknowledged. This project was also supported by the Sonderforschungsbereich 373 "Quantifikation und Simulation Ökonomischer Prozesse". JEL classification: F4, E32. 


\section{Introduction}

Over the past few years, we have witnessed a revival of models with multiple equilibria and sunspots that cause economic instability without having to rely on shocks to fundamentals. The work by Benhabib and Farmer $(1994,1996)$ and others has demonstrated that the occurrence of indeterminacy is not restricted to unrealistic special cases but that this phenomenon can occur in (representative agent versions of) dynamic general equilibrium models once the assumption of perfect markets is abandoned.

The intent of this paper is to develop a two sector open economy model with externalities in production. The model is therefore similar in its construction to Benhabib and Farmer's (1996) model of a closed economy. However, it will be shown that in the open economy version indeterminacy is obtained not only at lower returns to scale than in the closed economy case but also at insignificant levels thereof. This aspect of the model is of importance since recent empirical work has demonstrated that aggregate scale economies are close to constant. In particular, these estimates point to values that are too low to give a number of existing indeterminacy models a sufficient empirical foundation. ${ }^{1}$ Furthermore, indeterminacy does not require an elastic labor supply behavior. The reason for this characteristic in the present model is that perfect capital markets allow the smoothing of consumption via international lending and borrowing. Thus, the reduced form of the model becomes similar to the closed economy version with linear utility. This absence of curvature makes the construction of alternative investment paths available without the need to curtail consumption. ${ }^{2}$ Thus, indeterminacy arises from a correct sequence of price effects in the presence of externalities. These may well be minimal in size since, unlike in the closed economy variant, the competing consumption smoothing effect must not be offset.

Lahiri (1998) established that in a small open economy endogenous growth model, indeterminacy arises more straightforwardly than in closed economy versions. Jeon (1998) shows that in a one sector model with two kinds of capital (traded and nontraded capital goods), indeterminacy arises at lower increasing returns to scale than in Benhabib and Farmer's (1994) one sec-

\footnotetext{
${ }^{1}$ See for example Basu and Fernald (1997), Burnside (1995) and Harrison (1998a).

${ }^{2}$ See also Benhabib and Nishimura $(1996,1997)$.
} 
tor model. Weder (1998b) reports a similar result in a two sector general equilibrium model that also allows the endogenous choice of leisure.

The remainder of this paper is organized as follows. Section 2 presents the model. The equilibrium dynamics and indeterminacy are discussed in Section 3. An economic interpretation of the main result is offered in Section 4. Section 5 concludes.

\section{Model}

The model is based on the Benhabib and Farmer (1996) two sector optimal growth economy. The novel aspect here is that agents are allowed to borrow and lend internationally. We assume that the economy is too small to affect the world interest rate. Hence, the interest rate facing the country is parametric. Households are represented by a single agent that maximizes lifetime utility. The agent owns the stock of capital and receives income from wages and the rental of capital services to the firm sector. Firms produce either the consumption good or the investment good. Each firm has access to an externally increasing (or decreasing) returns to scale technology. All markets are perfectly competitive and factors of production are completely mobile between the economy's sectors. Consumer goods are tradeable whereas it is assumed that capital goods are nontradeables. ${ }^{3}$

\subsection{Technology}

The production technology of a typical firm in the investment good sector is $^{4}$

$$
y_{t}^{I}=\left[\left(K_{t}^{I}\right)^{\alpha}\left(L_{t}^{I}\right)^{1-\alpha}\right]^{\theta_{I}}\left[K_{t}^{\alpha} L_{t}^{1-\alpha}\right]^{\sigma_{I}}\left(k_{t}^{I}\right)^{\alpha}\left(l_{t}^{I}\right)^{1-\alpha} \quad \alpha \in(0,1)
$$

while that of the typical producer of the consumption good is given by

$$
y_{t}^{C}=\left[\left(K_{t}^{C}\right)^{\alpha}\left(L_{t}^{C}\right)^{1-\alpha}\right]^{\theta_{C}}\left[K_{t}^{\alpha} L_{t}^{1-\alpha}\right]^{\sigma_{C}}\left(k_{t}^{C}\right)^{\alpha}\left(l_{t}^{C}\right)^{1-\alpha} .
$$

Here $k_{t}^{I}$ and $l_{t}^{I}$ denote the capital and labor services used by the individual firm in the investment good producing sector. $K_{t}^{I}$ is the average stock of

\footnotetext{
${ }^{3}$ The dynamics of the model are principally unaffected as long as there is at least one nontradable investment good.

${ }^{4}$ The number of firms in each sector is unity.
} 
capital in this sector, $K_{t}$ stands for the stock of capital in the country. $\theta_{I}$ indicates sector-specific externalities and $\sigma_{I}$ denotes the degree of economy-wide externalities for the $I$-branch of the firms. The remaining variables are defined respectively and the index $C$ denotes the consumption good producing sector. We assume that the tradeable consumption good is the numeraire in symmetric equilibrium. Let by $w_{t}, r_{t}$ and $p_{t}$ denote the wage rate, the capital rental rate and the relative price of investment goods. In symmetric competitive equilibrium, the firms will hire capital and labor to satisfy the equalities:

$$
\begin{gathered}
w_{t}=(1-\alpha) p_{t} \kappa_{t}^{\theta_{I}}\left[K_{t}^{\alpha} L_{t}^{1-\alpha}\right]^{1+\theta_{I}+\sigma_{I}} L_{t}^{-1} \\
w_{t}=(1-\alpha)\left(1-\kappa_{t}\right)^{\theta_{C}}\left[K_{t}^{\alpha} L_{t}^{1-\alpha}\right]^{1+\theta_{C}+\sigma_{C}} L_{t}^{-1} \\
r_{t}=\alpha p_{t} \kappa_{t}^{\theta_{I}}\left[K_{t}^{\alpha} L_{t}^{1-\alpha}\right]^{1+\theta_{I}+\sigma_{I}} K_{t}^{-1} \\
r_{t}=\alpha\left(1-\kappa_{t}\right)^{\theta_{C}}\left[K_{t}^{\alpha} L_{t}^{1-\alpha}\right]^{1+\theta_{C}+\sigma_{C}} K_{t}^{-1} .
\end{gathered}
$$

In (3)-(6), we already have used the definition for the relative factor intensities

$$
\kappa_{t} \equiv \frac{K_{t}^{I}}{K_{t}}=\frac{L_{t}^{I}}{L_{t}} \quad \text { and } \quad 1-\kappa_{t} \equiv \frac{K_{t}^{C}}{K_{t}}=\frac{L_{t}^{C}}{L_{t}} .
$$

These equations can be combined to yield the relative price

$$
p_{t}=\left(1-\kappa_{t}\right)^{\theta_{C}} \kappa_{t}^{-\theta_{I}}\left[K_{t}^{\alpha} L_{t}^{1-\alpha}\right]^{\theta_{C}+\sigma_{C}-\theta_{I}-\sigma_{I}} .
$$

Further, output in the two sectors simplifies to

$$
Y_{t}^{I}=\kappa_{t}^{1+\theta_{I}}\left[K_{t}^{\alpha} L_{t}^{1-\alpha}\right]^{1+\theta_{I}+\sigma_{I}}
$$

and

$$
Y_{t}^{C}=\left(1-\kappa_{t}\right)^{1+\theta_{C}}\left[K_{t}^{\alpha} L_{t}^{1-\alpha}\right]^{1+\theta_{C}+\sigma_{C}}
$$

\section{$2.2 \quad$ Preferences}

The representative agent has preferences over sequences of consumption given by 


$$
U_{0}=\int_{0}^{\infty} U\left(C_{t}\right) e^{-\rho t} d t
$$

where $C_{t}$, and $\rho>0$ stand for consumption and the time discount factor. $U\left(C_{t}\right)$ has the usual properties. We normalize the fixed labor supply to unity. The representative individual owns the capital stock and lends its services to the firms. The agent's intertemporal budget constraint dictates

$$
\dot{D}_{t}=\varrho D_{t}+C_{t}+p_{t} I_{t}-w_{t}-r_{t} K_{t}
$$

where $D_{t}$ is the amount of foreign debt and $\varrho$ is the world interest rate. ${ }^{5}$ We further impose the standard simplifying assumption for small open economy models that the external interest rate is stationary and equal to the utility discount rate: $r_{t}-\delta=\varrho=\rho$. The capital accumulation technology is given by

$$
\dot{K}_{t}=I_{t}-\delta K_{t}
$$

with $\delta>0$ the rate of capital depreciation. We can analyze the agent's optimization problem by formulating the Hamiltonian

$$
\mathcal{H}_{t}=\left[U\left(C_{t}\right)+\lambda_{t}\left(w_{t}+r_{t} K_{t}-\rho D_{t}-C_{t}-p_{t} I_{t}\right)+\Lambda_{t}\left(I_{t}-\delta K_{t}\right)\right] e^{-\rho t}
$$

where $\Lambda_{t}$ and $\lambda_{t}$ are the shadow prices associated with the two constraints (11) and (12). The first order conditions are

$$
\begin{gathered}
U^{\prime}\left(C_{t}\right)=\lambda_{t} \\
\Lambda_{t}=\lambda_{t} p_{t} \\
\dot{\lambda}_{t}-\rho \lambda_{t}=-\rho \lambda_{t}
\end{gathered}
$$

${ }^{5}$ Note that the deficit on the current account is given by

$$
\dot{D}_{t}=\varrho D_{t}+C_{t}-Y_{t}^{C}
$$

This equation can be combined with the GDP income accounting relation

$$
w_{t}+r_{t} K_{t}=Y_{t}^{C}+p_{t} Y_{t}^{I}
$$

to yield (11). Furthermore $Y_{t}^{I}=I_{t}$. 


$$
\begin{gathered}
\dot{\Lambda}_{t}-\rho \Lambda_{t}=-r_{t} \lambda_{t}+\delta \Lambda_{t} \\
\lim _{t \rightarrow \infty}-\lambda_{t} D_{t} e^{-\rho t}=0 \\
\lim _{t \rightarrow \infty} \Lambda_{t} p_{t} K_{t} e^{-\rho t}=0 .
\end{gathered}
$$

(16) implies $\dot{\lambda}_{t}=0$, thus the constancy of consumption along the optimal path. This is a standard result in small open economy versions of the Ramsey growth model. ${ }^{6}$ It implies that complete consumption smoothing is realized through international lending and borrowing. Furthermore, since equation (14) drops out of the system, the curvature of the utility function has no influence on the model's dynamics.

\section{Equilibrium dynamics}

Since the model is recursive, the dynamics of consumption, relative price and debt can be determined once capital and the shadow price are determined.

\subsection{Dynamics}

Let us define $\mu_{t} \equiv \ln \Lambda_{t}$ and $k_{t} \equiv \ln K_{t}$. The model reduces to (12), (17), and (15) which can be transformed to

$$
\begin{gathered}
\dot{k}_{t}=e^{\left(1+\theta_{I}\right) \ln \kappa_{t}+\left(\alpha\left(1+\theta_{I}+\sigma_{I}\right)-1\right) k_{t}}-\delta \\
\dot{\mu}_{t}=(\delta+\rho)-\alpha e^{\theta_{I} \ln \kappa_{t}+\left(\alpha\left(1+\theta_{I}+\sigma_{I}\right)-1\right) k_{t}}
\end{gathered}
$$

and the static equation

$$
e^{\mu_{t}}=\lambda\left(1-e^{\ln \kappa_{t}}\right)^{\theta_{C}} e^{-\theta_{I} \ln \kappa_{t}+\alpha\left(\theta_{C}+\sigma_{C}-\theta_{I}-\sigma_{I}\right) k_{t}} .
$$

The last equation allows the partial derivatives of $\ln \kappa_{t}$ with respect to $\mu_{t}$ and $k_{t}$ to be derived. The nonlinear system is then Taylor-approximated about the steady state

$$
\left[\begin{array}{c}
\dot{\mu}_{t} \\
\dot{k}_{t}
\end{array}\right]=\mathbf{J}\left[\begin{array}{c}
\mu_{t}-\mu \\
k_{t}-k
\end{array}\right],
$$

\footnotetext{
${ }^{6}$ See for example Blanchard and Fischer (1989).
} 
where the matrix $\mathbf{J}$ is given by ${ }^{7}$

$$
\mathbf{J} \equiv\left[\begin{array}{cc}
\frac{(\delta+\rho) \theta_{I}}{\theta_{I}+\theta_{C} \kappa /(1-\kappa)} & -(\delta+\rho)\left[\alpha\left(1+\theta_{I}+\sigma_{I}\right)-1+\frac{\alpha \theta_{I}\left(\theta_{C}+\sigma_{C}-\theta_{I}-\sigma_{I}\right)}{\theta_{I}+\theta_{C} \kappa /(1-\kappa)}\right] \\
\frac{\delta\left(1+\theta_{I}\right)}{\theta_{I}+\theta_{C} \kappa /(1-\kappa)} & \delta\left[\alpha\left(1+\theta_{I}+\sigma_{I}\right)-1+\frac{\alpha\left(1+\theta_{I}\right)\left(\theta_{C}+\sigma_{C}-\theta_{I}-\sigma_{I}\right)}{\theta_{I}+\theta_{C} \kappa /(1-\kappa)}\right]
\end{array}\right] .
$$

Definition 1 (Perfect foresight equilibrium) In the model economy, a perfect foresight equilibrium is a set of sequences $\left\{k_{t}, \mu_{t}\right\}$ and an initial capital stock $k(0)>0$ satisfying (18) through (22).

Definition 2 (Indeterminacy) The equilibrium is indeterminate if there exists an infinite number of perfect foresight equilibrium sequences $\left\{k_{t}, \mu_{t}\right\}$.

$k_{t}$ is a predetermined variable and evolves continuously. The costate $\mu_{t}$ is a non-predetermined variable, that is, its initial value is not given by history and it may jump instantaneously in response to new information. The steady state is indeterminate if both eigenvalues of $\mathbf{J}$ are negative. ${ }^{8}$ In this case, the first order conditions and the transversality conditions are not sufficient to determine a unique solution. Multiplicity of this sort indicates that the rational expectations equilibria involve random variables that are unrelated to the economy's fundamentals simply because agents believe it to be so.

\subsection{Calibration}

Given the empirical character of the recent debate on the plausibility of indeterminacy models, a numerical solution seems advisable. However, even for analytical solutions, these parameter values give a guideline to rule out implausible parameter spaces. ${ }^{9}$ The following Table summarizes benchmark parameter values.

\footnotetext{
${ }^{7}$ The steady state $\kappa$ is given by

$$
\kappa=\frac{\alpha \delta}{\delta+\rho}
$$

${ }^{8}$ The model is also indeterminate if both eigenvalues are positive. However, in this rather uninteresting case the model is unstable and transversality conditions are ultimately violated (unless the system does not settle onto some stable cycle).

${ }^{9}$ These results are, however, largely unaffected by the particular numerical choices.
} 


\begin{tabular}{|c|c|c|}
\hline \multicolumn{3}{|l|}{ Table 1} \\
\hline$\alpha$ & $\delta$ & $\rho$ \\
\hline 0.40 & 0.10 & 0.05 \\
\hline
\end{tabular}

These choices are standard in Real Business Cycle calibration. They imply a labor share of sixty percent, an annual capital depreciation rate of ten percent, an annual utility discount rate of five percent, a capital/output ratio of 2.6 and a consumption share on output of 75 percent. We further restrict $\theta_{I}, \sigma_{I}, \theta_{C}$, and $\sigma_{C}$ each not to exceed 0.10 which is in line with recent empirical work by Basu and Fernald (1997) as well as Harrison (1998a). The latter reports slightly negative values for $\theta_{C}$. Realistic capital share calibrations allow us to make the plausible assumptions:

Assumption $1 \quad \alpha\left(1+\theta_{I}+\sigma_{I}\right)<1$ and $\alpha\left(1+\theta_{I}\right)<1$.

Assumption 1 can be interpreted as restricting (positive) externalities to be of modest size only.

\subsection{Indeterminacy and scale economies}

In this section the size of returns to scale that is needed to generate indeterminacy will be analyzed. If all four externalities are zero, the economy collapses to the one sector model, the equilibrium is unique and the dynamics are degenerate. Let us first consider the case of sector specific externalities only.

Indeterminacy when $\boldsymbol{\sigma}_{C}=\boldsymbol{\sigma}_{I}=\mathbf{0}$ We start with the parameter configuration that has been subjected to some recent empirical analysis and empirical confirmation: the presence of sector specific externalities only. The consumption good sector potentially exhibits scale diseconomies (see for example Harrison, 1998a, or Basu and Fernald, 1998).

When $\boldsymbol{\sigma}_{C}=\boldsymbol{\sigma}_{I}=\mathbf{0}$, the trace of the Jacobian $\mathbf{J}$ is given by

$$
\operatorname{tr} \mathbf{J}=\frac{\alpha \delta \rho \theta_{C}+\rho \theta_{I}(\rho+(1-\alpha) \delta)+\alpha \delta \theta_{C} \theta_{I}(\rho+\delta)}{\alpha \delta \theta_{C}+\delta \theta_{I}-\alpha}
$$

while its determinant is

$$
\operatorname{Det} \mathbf{J}=\frac{\delta(\rho+(1-\alpha) \delta)(\delta+\rho)\left(1-\left(1+\theta_{I}\right) \alpha\right)}{\alpha \delta \theta_{C}+(\rho+(1-\alpha) \delta) \theta_{I}} .
$$




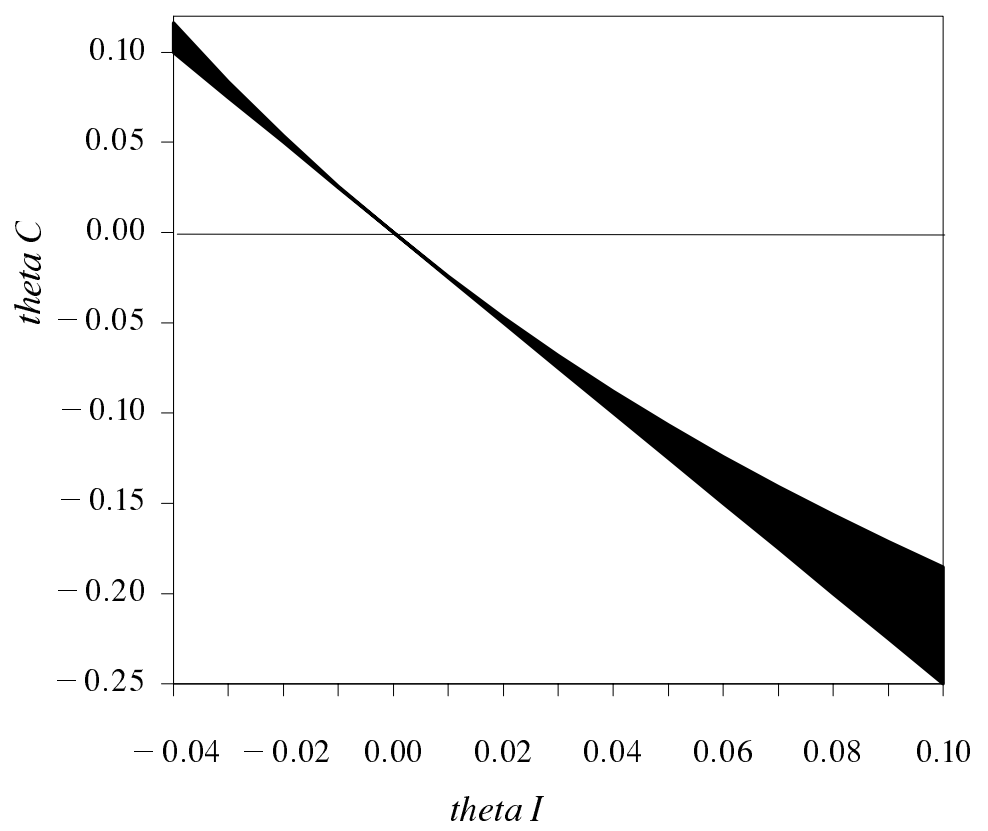

Figure 1: Indeterminacy with sector specific externalities.

Figure 1 reports the topological cases for nonzero $\left(\theta_{C}, \theta_{I}\right)$ combinations given the calibration in Table $1 .^{10}$ The dark cone indicates stable equilibria. Above this region, the model is unstable. Below the cone, the dynamics are of saddletype, thus, the model is determinate. Let us further investigate the indeterminacy conditions. ${ }^{11}$ From Assumption 1 , the numerator in $\operatorname{Det} \mathbf{J}$ is

\footnotetext{
${ }^{10}$ Different calibrations than the ones in Table 1 do not alter the qualitative results, rather only change the exact position of the three toplogical regimes.

${ }^{11}$ Stable (unstable) equilibria, thus two eigenvalues with negative (positive) real parts, are associated with a positive determinant and a negative (positive) trace. The eigenvalues split around zero, e.g. a saddle, if the determinant is negative.
} 
always positive. Thus, nonsaddlepoint behavior occurs for ${ }^{12}$

$$
\theta_{C}>\left(\frac{\rho+(1-\alpha) \delta}{\alpha \delta}\right) \theta_{I}
$$

A necessary condition for multiplicity is that the sector specific externalities are of opposite sign. From Figure 1, we can also see that as $\theta_{C}$ increases, the dynamics change from a sink (the standard indeterminacy classification) to a source. In other words, there exists a function $\theta_{C}^{*}=\theta_{C}^{*}\left(\theta_{I}^{*}\right)$ such that the equilibrium has a simple pair of imaginary eigenvalues and a Hopf bifurcation takes place. In this parametric neighborhood, the equilibrium is stable for $\theta_{C}<\theta_{C}^{*}$ and unstable for $\theta_{C}>\theta_{C}^{*}$. In particular, the trace vanishes when

$$
\theta_{I}^{*}=\frac{\alpha \delta \rho}{-\rho(\rho+(1-\alpha) \delta) \theta_{C}^{*-1}-\alpha \delta \rho-\alpha \delta^{2}} .
$$

Thus, by continuity, if the negative (positive) externality parameter $\theta_{C}$ is infinitesimally close to zero, a positive (negative) value for $\theta_{I}$ will always exists such that indeterminacy is possible: ${ }^{13}$ indeterminacy arises at virtually zero departures from the constant returns to scale case. Moreover, the equilibrium becomes a saddle along the straight line $\theta_{C}^{\text {saddle }}=-\frac{\rho+(1-\alpha) \delta}{\alpha \delta} \theta_{I}^{\text {saddle }}$. At this bifurcation point, one eigenvalue passes through minus infinity. Therefore, indeterminacy is satisfied close to the bifurcation boundary. Finally, it should be noted that given the results by Harrison (1998a), it is plausible to restrict $\theta_{C}<\theta_{I}$. In this case the model's average externalities are negative in the indeterminacy region. In the closed economy variant of two sector model with sector specific externalities indeterminacy is obtained at $\theta_{I}>1.07$. $^{14}$ The following main Proposition of the paper summarizes the results.

Proposition 1 The small open economy two sector model with sector specific externalities generates indeterminacy at virtually constant returns to scale. For plausible parameter constellations, the overall economy exhibits decreasing returns to scale.

For additional understanding of the indeterminacy mechanism, consider the following two special cases.

\footnotetext{
${ }^{12}$ This is equivalent to $\partial \ln \kappa_{t} / \partial \mu_{t}<0$.

${ }^{13}$ Recall that at $\theta_{C}=\theta_{I}=0$ the model must be determinate, hence, the origin itself is not included. Numerical calibrations find indeterminacy at $\theta_{I} \approx 0.001$.

${ }^{14}$ Harrison (1998b) and Weder (1998c) show that it is the increasing returns in the investment sector that drive the result.
} 
Dynamics when $\theta_{C}=\boldsymbol{\sigma}_{C}=\boldsymbol{\sigma}_{I}=\mathbf{0}$ In this case, the trace of $\mathbf{J}$ becomes $\rho>0$, thus, the steady state can never be a sink. The determinant is given by

$$
\operatorname{Det} \mathbf{J}=\frac{\delta(\rho+\delta)\left(1-\left(1+\theta_{I}\right) \alpha\right)}{\theta_{I}}
$$

Given assumption 1, sign $\operatorname{Det} \mathbf{J}=\operatorname{sign} \theta_{I}$. Hence, positive sectoral externalities in the investment sector imply that the steady state is a source. Otherwise the equilibrium is a saddle point and is unique.

Dynamics when $\theta_{I}=\boldsymbol{\sigma}_{C}=\boldsymbol{\sigma}_{I}=\mathbf{0}$ In this case, the trace of $\mathbf{J}$ becomes $\rho>0$ again. The determinant is given by

$$
\operatorname{Det} \mathbf{J}=\frac{(1-\alpha)(\rho+\delta)(\rho+(1-\alpha) \delta)}{\alpha \theta_{C}}
$$

It follows that sign Det $\mathbf{J}=\operatorname{sign} \theta_{C}$. Hence, positive sectoral externalities in the consumption good sector imply that the steady state is a source. Otherwise the equilibrium is a saddle point. The next Proposition restates the result.

Proposition 2 For indeterminacy to occur, sector specific externalities must be present in both sectors. Moreover, sector specific diseconomies of some sort are necessary for nonsaddle equilibria to be stable.

Dynamics when $\theta_{C}=\boldsymbol{\sigma}_{C}=\mathbf{0}$ If departures from constant returns are limited to the investment sector, the trace if the matrix $\mathbf{J}$ becomes

$$
\operatorname{tr} \mathbf{J}=\rho-\frac{\alpha \delta \sigma_{I}}{\theta_{I}}
$$

and the determinant is given by

$$
\operatorname{Det} \mathbf{J}=\frac{\delta(\rho+\delta)\left(1-\left(1+\theta_{I}+\sigma_{I}\right) \alpha\right)}{\theta_{I}} .
$$

Given Assumption 1, a necessary condition for indeterminacy is $\theta_{I}>0$, otherwise the determinant of the Jacobian is negative. Further, the trace is negative for

$$
\theta_{I}<\frac{\alpha \delta}{\rho} \sigma_{I}
$$

The following proposition summarizes the result. 
Proposition 3 Suppose the production function in the consumption good sector is constant returns to scale. Indeterminacy requires positive sector specific and positive aggregate externalities in the investment goods producing sector. The steady state is a sink if $\theta_{I} / \sigma_{I}<\alpha \delta / \rho$.

Dynamics when $\theta_{I}=\boldsymbol{\sigma}_{I}=\mathbf{0}$ If the investment good sector operates under constant returns and externalities are present in the consumption good sector, the trace and determinant become

$$
\operatorname{tr} \mathbf{J}=\frac{\sigma_{C}(\rho+(1-\alpha) \delta)+\rho \theta_{C}}{\theta_{C}}
$$

and

$$
\operatorname{Det} \mathbf{J}=\frac{(1-\alpha)(\rho+\delta)(\rho+(1-\alpha) \delta)}{\alpha \theta_{C}} .
$$

A positive determinant is given for all $\theta_{C}>0$. Indeterminacy is obtained for

$$
\sigma_{C}<-\frac{\rho+(1-\alpha) \delta}{\rho} \theta_{C}
$$

Thus, a stable steady state requires negative aggregate externalities. Let us summarize these results.

Proposition 4 Suppose the production function in the investment good sector is constant returns to scale. Indeterminacy requires positive sector specific and negative aggregate externalities in the consumption goods producing sector. The steady state is a sink if $\sigma_{C} / \theta_{C}<(1-\alpha) \delta / \rho-1$.

\section{Interpretation}

It has been shown in the preceding section that indeterminacy can be obtained at plausible parameter values. The mechanism that leads to indeterminacy differs somewhat to that in the closed economy model. Let us consider the discrete time version of the model. The intertemporal Euler equation is given by

$$
\lambda_{t} p_{t}=\frac{\lambda_{t+1}}{1+\rho}\left[r_{t+1}+(1-\delta) p_{t+1}\right]
$$


If agents believe in realizing capital gains by reallocating resources over time, they will start investing today and purchase more consumption goods in the future. If returns to scale in the investment good sector are sufficiently high, the relative price will first fall (as a result of the sector-specific positive externalities) and then rise. ${ }^{15}$ The expected price path is self-fulfilling. This is clear since, as a result of this reallocation, both $\lambda_{t}$ and $p_{t}$ move in opposite direction. Thus, the effect of increasing returns on relative prices must be sufficiently strong to overcome the agent's desire to smooth consumption. Furthermore, Harrison (1998b) and Weder (1998a, 1998c) show that it is generally sufficient to assume increasing returns in the investment good producing sector only.

Unlike the Benhabib and Farmer (1996) setup, in the small open economy there are no costs of foregone consumption along alternative paths. Perfect international capital markets allow the perfect smoothing of consumption: the shadow price of wealth $\lambda$ is a constant. Therefore, if agents want to increase investment, the realization of consumption is unaffected. Even when utility has curvature alternative equilibria can easily be constructed. ${ }^{16}$ The reduced form of the model becomes similar to the closed economy version with linear utility and only minor departures from constant returns to scale are needed to produce the self-fulfilling price dynamics. Indeterminacy eventuates from an appropriate sequence of price effects that arise in the presence of externalities. In a small open economy model such as the present, the externalities must be only minimal in size to initiate this price sequence. Thus, indeterminacy is obtained at much lower values than in the closed economy variant. However, if sector specific externalities are considered only, indeterminacy requires that the model must possess some form of a dampening effect similar to the Howitt and McAfee (1988) search externality model. ${ }^{17}$ Unless this negative effect is present, capital gains could be forwarded ad infinitum and the economy would eventually depart from its steady state equilibrium

\footnotetext{
${ }^{15}$ It can be shown that the production possibility frontier is convex as long as there is increasing returns in at least one sector (see Harrison, 1998b).

${ }^{16}$ In the closed economy version an investment boom requires an outward shift of labor supply to be able to produce more of the investment good while at the same time satisfying consumption smoothing. This mechanism is completely absent here.

${ }^{17}$ Howitt and McAfee (1988) have two externalities (one postive, one negative) in their model. The authors actually build on result known from Liviatan and Samuelson (1969) that stationary states are either saddles or unstable.
} 
forever. Recall the discrete time Euler equation: if agents increase their demand for investment goods, the relative price falls as a consequence of the positive sector-specific externalities. There is no mechanism that checks the price dynamics since $\lambda$ is constant. In the continuous time case of the small open economy, (17) is the relevant Euler equation. The curvature of utility is no longer an argument. When investment increases, $p_{t}$ falls as a result of the positive sector specific externalities. Intertemporal equilibrium now requires that the shadow price of capital appreciates. However, the price fall of capital induces further investment activity, hence, the process would apply over and over again. To reverse the infinite appreciation and to rule out an ultimate violation of the transversality conditions, some form of negative externalities must be set in motion. This is the result of Proposition 1. Thus, intertemporal equilibrium eventually requires a depreciation and the economy moves back towards the steady state, giving rise to an alternative equilibrium trajectory.

\section{Summary}

This paper presents a small open economy version of the Benhabib and Farmer (1996) two sector optimal growth model with sector specific externalities. It is shown that indeterminacy is considerably easier to obtain under this regime without perfect world capital markets than in the closed economy variant. Furthermore the result is not dependent on a high labor supply elasticity. Although the model is highly stylized, it clearly gives an indication that opening up international capital markets can be destabilizing. More generally, it has been shown that the two sector small open economy versions of the optimal growth model have less degenerate dynamics than its one sector relatives.

\section{References}

[1] Basu, Susanto and John G. Fernald (1997): "Returns to Scale in U.S. Production: Estimates and Implications", Journal of Political Economy 105, 249-283. 
[2] Benhabib, Jess and Roger E. A. Farmer (1994): "Indeterminacy and Increasing Returns", Journal of Economic Theory 61, 19-41.

[3] Benhabib, Jess and Roger E. A. Farmer (1996): "Indeterminacy and Sector-Specific Externalities", Journal of Monetary Economics 37, 421443.

[4] Benhabib, Jess and Kazuo Nishimura (1997): "Indeterminacy Under Constant Returns to Scale", Department of Economics New York University, mimeo.

[5] Benhabib, Jess and Kazuo Nishimura (1996): "Indeterminacy and Sunspots with Constant Returns to Scale", C. V. Starr Center for Applied Economics Economic Research Report \#96-44.

[6] Blanchard, Olivier J. and Stanley Fischer (1989): Lectures on Macroeconomics, MIT Press, Cambridge.

[7] Burnside, Craig (1996): "Production Function Regressions, Returns to Scale and Externalities", Journal of Monetary Economics 37, 177-201.

[8] Guckenheimer, John and Philip Holmes (1983): Nonlinear Oscillations, Dynamical Systems, and Bifurcations of Vector Fields, Springer-Verlag, New York.

[9] Harrison, Sharon G. (1998a): "Production Externalities and Indeterminacy in a Two Sector Model: Theory and Evidence", Department of Economics Barnard College, Working Paper \#98-05.

[10] Harrison, Sharon G. (1998b): "Indeterminacy in a Model with Sector Specific Externalities", Department of Economics Barnard College, Working Paper \#98-07.

[11] Howitt, Peter and R. Preston McAfee (1988): "Stability of Equilibria with Externalities", Quarterly Journal of Economics 103, 261-277.

[12] Jeon, Jongkyou (1998): "Indeterminacy and Business Cycles in a Small Open Economy", Department of Economics UCLA, mimeo.

[13] Kuznetsov, Yuri A. (1998): Elements of Applied Bifurcation Theory, Springer-Verlag, New York. 
[14] Lahiri, Amartya (1998): "Growth and Equilibrium Indeterminacy: The role of capital mobility", Department of Economics UCLA, mimeo.

[15] Liviatan, Nissan and Paul A. Samuelson (1969): "Notes on Turnpikes: Stable and Unstable", Journal of Economic Theory 1, 454-475.

[16] Weder, Mark (1998a): "Fickle Consumers, Durable Goods and Business Cycles", Journal of Economic Theory 81, 37-57.

[17] Weder, Mark (1998b): "Indeterminacy in a Two Sector Small Open Economy Optimal Growth Model", Department of Economics Humboldt University Berlin, mimeo.

[18] Weder, Mark (1998c): "Animal Spirits, Technology Shocks and the Business Cycle", Journal of Economic Dynamics and Control, (forthcoming). 